\title{
Employee Job Class
}

National Cancer Institute

\section{Source}

National Cancer Institute. Employee Job Class. NCI Thesaurus. Code C74565.

A coding system used to specify attributes of an occupation. 